\title{
Architecture for Efficient Monitoring and Management of Sensor Networks
}

\author{
Mohamed Younis ${ }^{1}$, Poonam Munshi ${ }^{1}$, and Ehab Al-Shaer ${ }^{2}$ \\ ${ }^{1}$ Dept. of Computer Science and Elect. Eng. \\ University of Maryland Baltimore County \\ Baltimore, MD 21250 \\ \{younis, poonam1\}@cs.umbc.edu \\ ${ }^{2}$ School of Computer Sc.,Telecom. and Info. Sys. \\ DePaul University \\ Chicago, IL 60604 \\ ehab@cs.depaul.edu
}

\begin{abstract}
Wireless sensor networks are poised to increase the efficiency of many military and civil applications, such as disaster management. Typically sensors collect data about their surrounding and forward that data to a command center, either directly or through a base-station. Due to inhospitable conditions, these sensors are not always deployed uniformly in an area of interest and some sensors can be unreachable because they are too distant from the base-station or simply because there exist obstacles in their path. This paper focuses on reducing the sensitivity of the operation and monitoring of sensor networks to the ambiguity of the propagation model of the radio signal. We define 'agent' sensors, which monitor the health and relay messages to and from unreachable sensors. We form groups of sensors around these agents while considering the load on each agent. An energy-aware routing of data collected by and relayed by these agents is performed. Our approach localizes communication which reduces the amount of sensor energy expended in transmission, enables efficient monitoring of sensor resources and health status and allows optimal management of deployed sensors for increased network lifetime. The approach is validated by the simulation results.
\end{abstract}

Keywords: Sensor networks, Energy efficient design, Network monitoring, Energy-aware communication

\section{Introduction}

Advances in microelectronics have enabled the development of small sensing devices equipped with signal processing and wireless communication capabilities. Such sensors are usually deployed in an ad-hoc manner in the area of interest to track events and gather data about the environment. Networking unattended sensors is expected to have significant impact on the efficiency of many military and civil applications, such as combat field surveillance, security and disaster management12345. Sensors in such applications are typically disposable and expected to last until their energy drains. Therefore, energy is a very scarce resource for such sensor systems and has to be 
managed wisely in order to extend the life of the sensors for the duration of a particular mission.

Sensors probe the surrounding environment and generate reports of the collected readings. Such reports are sent via the radio transmitter to a command center, usually through a gateway, deployed in the physical proximity of the sensors (Fig. 1). The gateway aggregates and analyzes the

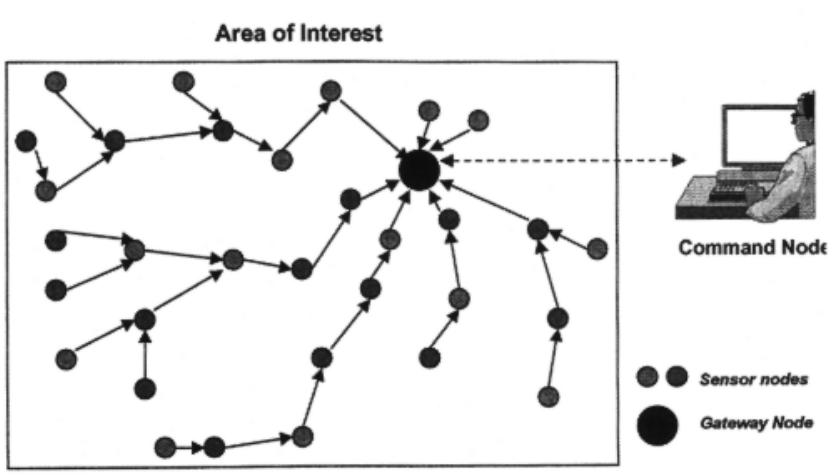

Fig. 1. Three-tier sensor network architecture sensed data prior to forwarding to the command center. The gateway can perform fusion of the sensed data in order to filter out erroneous data and anomalies and to draw conclusions from the reported data over a period of time. For example, in a reconnaissance-oriented sensor network, sensor data indicates detection of a target while fusion of multiple sensor reports can be used for tracking and identifying the detected target 6 .

Signal processing and communication activities are the main consumers of sensor's energy. Since sensors are battery-operated, keeping the sensor active all the time will limit the duration that the battery can last. Therefore, optimal organization and management of the sensor network are very crucial in order to perform the desired function with an acceptable level of quality and to maintain sufficient sensors' energy to last for the duration of the required mission. On the other hand effective operation of sensor networks requires knowledge of the status of available sensors. Based on the remaining energy, field of view and communication range the gateway can assess sensor coverage and balance the load on the sensors in order to extend the life of the network. Therefore, the gateway needs to monitor the health status and remaining energy of the sensors. Monitoring the sensor network is a challenging problem due to the resource limitation and the large network size. Monitoring protocols for wired networks introduce large overhead and are thus deemed unsuitable for such resource constrained sensor nodes.

In order to minimize communication energy, multi-hop routes are usually employed for collecting sensor's reports. Transmission power of radio circuits is generally proportional to distance squared or even higher order for environment rich with obstacles and interference sources. Multi-hop paths minimize the total transmission power by shortening the distance a radio signal needs to travel from a transmitter to a receiver. The sensors are ideally able to communicate with the gateway through shorthaul communication under all circumstances either directly or by using multiple hops. This type of model assumes that the gateway is directly reachable to all sensors under all conditions. However, sensor deployment can be non-uniform due to inhospitable conditions and the terrain may not always be low. In fact, most practical deployment scenarios involve regions that have various obstacles (e.g. buildings, trees, etc.) pre- 
venting effective signal propagation. This makes the sensor network sensitive to the propagation model. Consider for example the two cases depicted in figures $2 \mathrm{a}$ and $2 \mathrm{~b}$. In Fig. 2a, the sensors and gateway are within the communication range of each other. However the gateway is not able to directly communicate with some sensors due to an obstacle on the communication path between them. Another possible case is shown in Fig. 2b where some sensors are not directly reachable to the gateway because they are located too far from the gateway.

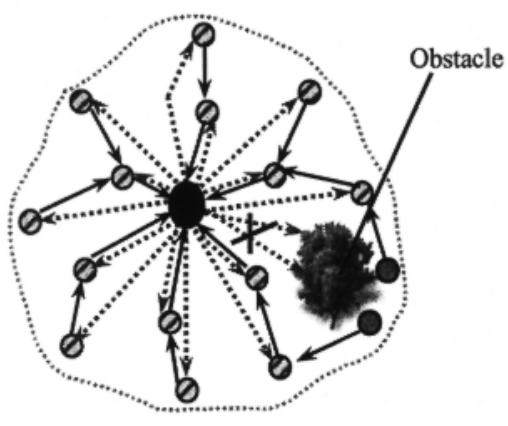

Fig. 2. a) Some sensors are not reachable by the gateway due to an obstacle between them

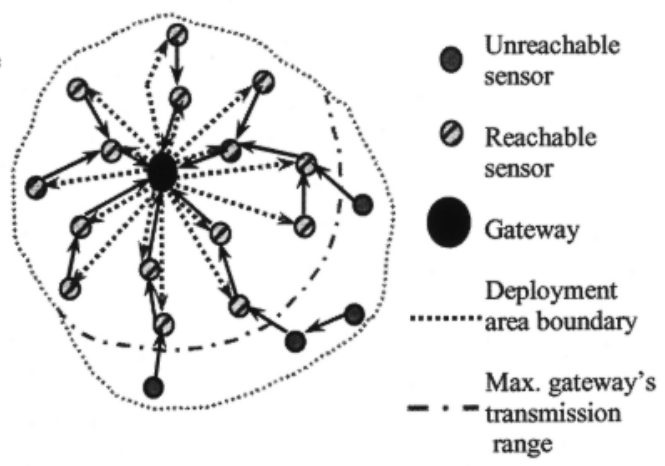

Fig. 2. b) Some sensors lie outside the transmission range of the gateway

In this paper, our main objective is to reduce the sensitivity of the operation of the sensor network to the ambiguity of the propagation model and to enable effective monitoring of sensors status. We introduce an additional tier in the network by forming groups of sensors. An agent sensor is designated for each group of sensors that cannot be directly reached by the gateway. Agents relay commands from the gateway, report the status of group members to the gateway and forward collected reports within the group to next assigned hops. We present an efficient technique for sensor grouping that considers sensor proximity and reachability to the gateway while balancing load on picked agent sensors. An energy-aware multi-hop routing is further employed among agent sensors to minimize communication energy. Our approach localizes communication within the group and limits the effect of poor propagation of radio signals. Furthermore, sensor health and resource status can be efficiently monitored. While clustering and energy-conscious routing are widely pursued in sensor networks, we are not aware of other work that efficiently handle both monitoring and management of the network resources.

\subsection{System Model}

A set of sensors is spread throughout an area of interest to detect and track events/targets in this area. The sensors are battery-operated and are empowered with limited data processing engines. Their mission is dynamically changing to serve the need of a command center. A significantly less energy-constrained gateway node is 
deployed in the physical proximity of sensors. Both the sensor nodes and the gateway are stationary. The gateway is assumed to know the geographical location of deployed sensors. Sensors are discovered through repeated beacons [7]7. The gateway is responsible for organizing the activities at sensor nodes to achieve a mission, fusing collected data, coordinating communication traffic and interacting with the command node. The gateway node sends to the command node reports generated through fusion of sensor readings, e.g. tracks of detected targets. The command node presents these reports to the user and performs system-level fusion of the received reports for an overall situation awareness. The system architecture for the sensor network is depicted in Fig. 1.

The sensor is assumed to be capable of reporting its remaining energy and operating in an active mode or a low-power stand-by mode. The sensing and processing circuits can be powered on and off. In addition both the radio transmitter and receiver can be independently turned on and off and the transmission power can be programmed for a required range. It is worth noting that most of these capabilities are available on some of the advanced sensors, e.g. the Acoustic Ballistic Module from SenTech Inc. [9]. It is also assumed that the sensor can act as a relay to forward data from another sensor. We refer to the gateway's selection of a subset of the sensors for probing the environment as network organization and to the data routing and medium access arbitration as network management. In our architecture, network organization and management are energy aware and rely on the knowledge of energy reserve at each sensor. Network monitoring refers to the process of querying the health status of sensors and checking their remaining energy.

\subsection{Related Work}

Routing of sensor data has been one of the challenging areas in wireless sensor network research. It usually involves multi-hop communications and has been studied as part of the network layer problems 101112[13]. Despite the similarity between sensor and mobile ad-hoc networks, routing approaches for ad-hoc networks proved not to be suitable for sensors networks. This is due to different routing requirements for ad-hoc and sensor networks in several aspects. For instance, communication in sensor networks is from multiple sources to a single sink, which is not the case in ad-hoc networks. Moreover, there is a major energy resource constraint for the sensor nodes. As a consequence, many new algorithms have been proposed for the problem of routing data in sensor networks. These routing mechanisms can be classified as data-centric [10], hierarchical 10 or location-based 12. Although current research on routing of sensor data mostly focused on protocols that are energy aware to maximize the lifetime of the network, we are not aware on any approach the handle both energy-aware management and efficient monitoring of sensor status.

Achieving energy saving through activation of a limited subset of nodes in an adhoc wireless network has been the goal of some recent research such as SPAN [14], GAF 14 and ASCENT 15. Both SPAN and GAF are distributed approaches that require nodes in close proximity to arbitrate and activate the least number of nodes needed to ensure connectivity. Nodes that are not activated are allowed to switch to a low energy sleep mode. While GAF uses nodes' geographical location to form gridbased cluster of nodes, SPAN relies on local coordination among neighbors. In 
ASCENT, the decision for being active is the courtesy of the node In our approach node's state is determined at the gateway while considering processing duties in the sensor's state transition. In addition, we monitor sensor's status to enhance the effectiveness of network operation.

The unique requirements of monitoring sensor networks are well analyzed in [17]. An approach has been proposed that empowers neighbors to monitor each other. Although ideally neighbors should notify the network controller upon agreement on the faulty status of a particular sensor, they may not be able to reach the controller due to radio signal propagation problems. Instead such neighbors collaboratively handle the failure. Such model is different from ours and does not handle the core issue of sensor reachability. Another mechanism for distributed identification of faulty sensors is reported in 17. Sensors are assumed to be on all time and involved in a rather multiphase complex protocol. Instead of monitoring the individual sensor, the approach proposed in 18 track energy in small areas. Energy reserves are continuously scanned and aggregated locally and only a distribution of available energy on the network level is formed. Such approach requires sensors to be active all time and introduces additional aggregation at the sensor level.

\section{Multi-tier Sensor Network Architecture}

As discussed earlier, sensors are typically deployed randomly for probing the environment in a particular area of interest and transmit collected reports to a command center via radio. Since sensors are usually battery-operated, their energy has to be managed wisely. In addition, given the limited resources the sensor has, sensor status has to be closely watched in order to ensure a good coverage by enough functional sensors. We propose a multi-tier architecture for monitoring and management of sensor networks. A gateway node is to be deployed in the vicinity of sensors in order to organize the sensors according to the application requirements and to manage the collection of sensors data. To overcome weak propagation of radio signals caused by terrain and high level of signal interference and which prevents some sensors from receiving gateway messages, we form groups of sensors in close proximity with at least one reachable node.

For simplicity, we assume that the gateway can reach all deployed sensors in at most 2 hops. We consider all sensors directly reachable by the gateway as relays that can forward sensor's data to the gateway. From these relays we pick some 'agent' sensors, which act as communication hops for sensors that cannot receive gateway messages. We form groups of sensors around these agents while considering the processing and communication load on each agent. A group should have an agent, which will be responsible for interaction with gateway on behalf of that group(Fig. 3).

This approach guarantees higher level of node reachability for the gateway and reduces the energy consumed by the sensors in communication. The agent selection and grouping alleviates load on sensors for transmission by localizing the communication for the uplink. The sensors within the group need to transmit only to the agent. In addition, the selection of the path for the downlink from the gateway is more determi- 
nistic since the gateway needs to simply convey messages to the assigned agent who can then forward it to the recipient sensors within their group in 1-hop.

The next three subsections discuss network bootstrapping, group formation and finally energy-aware data routing and efficient monitoring of sensor's resources.
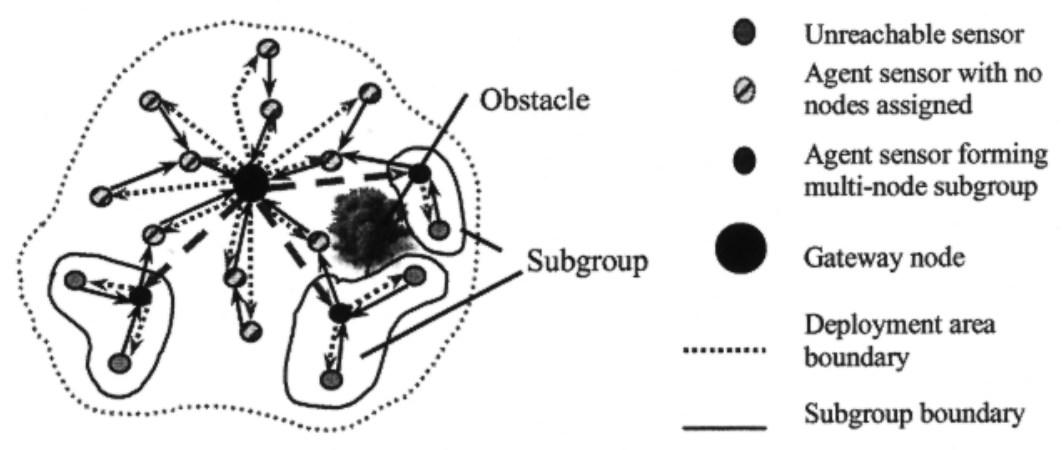

Fig. 3. A group can have only the agent or can include some unreachable sensors as well

\subsection{Network Bootstrapping}

Network bootstrapping refers to the initialization phase, in which the gateway finds out the location of the deployed sensors and the quality of the links among these sensors and between them and the gateway. Sensors discovery is assumed to be performed using repeated beacons, after which each sensor would know the set of sensors whose radio transmission can be heard 6. The gateway also makes multiple announcements to assess the quality of its links to the deployed sensors 7 . The gateway then probes the deployed sensors to query the status of the communication links. The gateway is less energy constrained as thus expected to possess a larger transmission range than sensors. Therefore, sensors whose transmission range covers the gateway convoy location of other sensors that cannot reach the gateway and whether they can hear the gateway announcement.

Let $S$ be the set of all deployed sensors with $|S|=n$. Let $S_{R}$ and $S_{U R}$ be the set of sensors that respectively can and cannot receive messages that are directly transmitted by the gateway. $S_{R}$ and $S_{U R}$ can be defined as follows:

$$
\mathrm{S}_{\mathrm{R}}=\left\{\mathrm{s}_{\mathrm{i}} \in S: \mathrm{G} \rightarrow \mathrm{s}_{\mathrm{i}} \text { for all } i\right\}, \quad \mathrm{S}_{\mathrm{UR}}=\left\{\mathrm{s}_{\mathrm{j}} \in S: \mathrm{G} \not \rightarrow \mathrm{s}_{\mathrm{j}} \text { for all } j\right\} .
$$

It is worth noting that $S_{R} \cup S_{U R}$ may not equal to $S$ since there can be some sensors whose transmission cannot be heard by any node, e.g. fell in a deep hole. At the end of the bootstrapping phase, the gateway establish for every sensor the following attributes:

- Gateway-link-status: a variable set to 0 or 1 depending whether the sensor belongs to $\mathrm{S}_{\mathrm{R}}$ or $\mathrm{S}_{\mathrm{UR}}$ respectively.

- Neighbor-list: all sensors, which can hear its transmission.

- Hop-list: all neighbors, which are in the set $\mathrm{S}_{\mathrm{R}}$. 
These attributes are used by the gateway to form groups of sensors as explained next.

\subsection{Group Formation}

Grouping of sensors is geared mainly for ensuring a connection between the gateway and sensors that belong to $S_{U R}$. The idea is to designate one or more sensors in $S_{R}$, called agents, to be responsible for convoying gateway messages to sensors in $S_{U R}$ and relaying data collected by these sensors to the gateway (Fig. 4). Our approach for group formation that balances the load among selected agents in order to maximize their life. To facilitate such hierarchy, the gateway adds another attribute called agentId for every sensor, to identify the agent node to which it is assigned. Sensors that belong to $S_{R}$ are assigned (agentId $=0$ ) since they do not need an agent to hear the gateway transmission. In addition, the gateway designates a group-list for every potential agent sensors to track members of its group. Group formation is performed as follows:

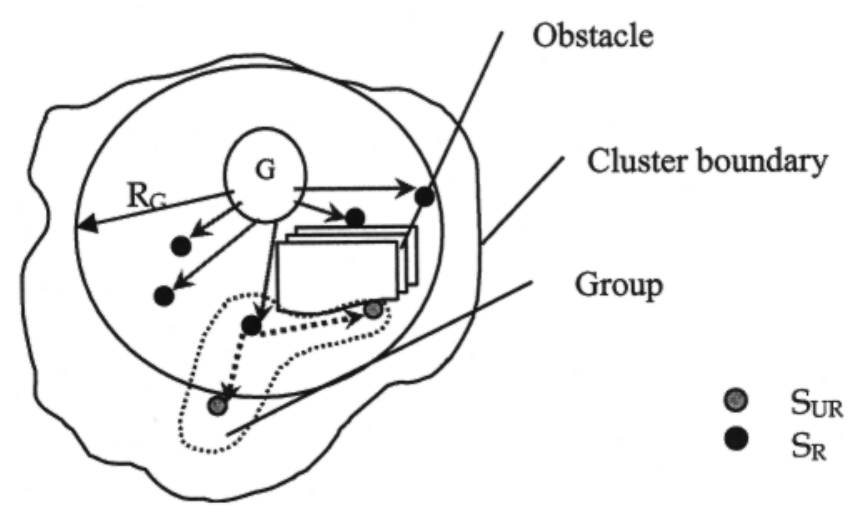

Fig. 4. Formation of groups by selection of 'agents' from the set of reachable sensors $S_{R}$.

Step 1: For each sensor $S_{j} \in S_{U R}$, the gateway calculates the Hop-list of sensors by picking neighbors that belong to the set $S_{R}$. Sensors in $S_{U R}$ are sorted in ascending order according to the cardinality of their Hop-list.

Step 2: Starting from the sensors with lowest cardinality of the hop-list, we assign an agent sensor sensors $S_{i}$ in $S_{R}$ for every sensor $S_{j} \in S_{U R}$. There are two cases:

CASE 1: $\mid$ Hop-list $\left(\mathrm{S}_{\mathrm{j}}\right) \mid=1 \quad$ // node has a link to only one sensor in $\mathrm{S}_{\mathrm{R}}$ Assign sensor $\mathrm{S}_{\mathrm{j}}$ to the only reachable sensor $\mathrm{S}_{\mathrm{i}}$ by making $\operatorname{agentId}\left(\mathrm{S}_{\mathrm{j}}\right)=\mathrm{S}_{\mathrm{i}}$. Add $\mathrm{S}_{\mathrm{j}}$ to group-list $\left(\mathrm{S}_{\mathrm{i}}\right)$.

CASE 2: $\mid$ Hop-list $\left(\mathrm{S}_{\mathrm{j}}\right) \mid>1 \quad$ // node has a link to multiple sensors in $\mathrm{S}_{\mathrm{R}}$

Calculate the assignment cost $(A C)$ for each sensor $S_{i}$ in the Hop-list of $S_{j}$.

Assign sensor $\mathrm{S}_{\mathrm{j}}$ to the sensor $\mathrm{S}_{\mathrm{k}}$ in $\operatorname{Hop}$ - list $\left(\mathrm{S}_{\mathrm{j}}\right)$ with least assignment cost $\left(\operatorname{agentId}\left(\mathrm{S}_{\mathrm{j}}\right)=\mathrm{S}_{\mathrm{k}}\right)$.

Add $\mathrm{S}_{\mathrm{j}}$ to group-list $\left(\mathrm{S}_{\mathrm{k}}\right)$.

For a node $S_{j} \in S_{U R}$, which can reach more than one sensor in $S_{R}$, we select the node that appears to be the best i.e. has least cost at the time of assignment. The cost is a quantification of two important factors reflecting the interest of both group mem- 
bers and the agent (group leader). Ideally a node $S_{j} \in S_{U R}$ is to be assigned to a group, whose agent is the closest to $S_{j}$ compared to other sensors in the Hop-list $\left(S_{j}\right)$ so that communication energy is minimized. Also, we would like to ensure that the load is balanced among agents to increase their lifetime and avoid repeating the grouping process very often to recover from an agent failure. The time for the first node to die is one of the most important performance metrics for sensor networks 112. We define the assignment cost (AC) as a linear combination of both factors as follows:

$$
\mathrm{AC}_{\mathrm{i}}=a_{1} \times \text { Communication cost on the link }\left(\mathrm{S}_{\mathrm{j}} \rightarrow \mathrm{S}_{\mathrm{i}}\right)+a_{2} \times\left|\operatorname{group}-\operatorname{list}\left(\mathrm{S}_{\mathrm{i}}\right)\right|
$$

The communication cost on the link $\left(\mathrm{S}_{\mathrm{j}} \rightarrow \mathrm{S}_{\mathrm{i}}\right)$ is calculated based on the distance between $S_{j}$ and $S_{i}$. The factors $a_{1}$ and $a_{2}$ are weighting constants that give us the flexibility of deciding which of the above two parameters is more important when forming groups. The load on sensor $\mathrm{S}_{\mathrm{i}}$ is directly proportional to the number of sensors assigned to it (including $\mathrm{S}_{\mathrm{j}}$ ), assuming that all sensors produce data at the same rate. It is worth noting that not all sensors of $S_{R}$ will be designated as a group leader. In this case they will be considered agents without group members in order to unify route setup. We next explain how sensor grouping enables efficient monitoring and data routing.

\subsection{Routing and Monitoring}

Since the gateway is significantly less energy-constrained compared to sensors, it is poised to efficiently manage the network. In our architecture, the gateway takes charge of sensor organization and network management based on the application mission and available energy in each sensor. Because the sensor is committed to data processing and communication, it is advantageous to offload management decision from the resource-constrained sensor nodes. In addition, since the gateway has a wide view of the network, the routing decisions should be simpler and more efficient than decisions based on local views at the sensor level. Moreover, knowledge of networkwide sensor status enhances the robustness and effectiveness of media access control because the decision to turn a node receiver off will be more accurate and deterministic than a decision based on a local MAC protocol 1920.

Sensor organization enables the activation of only a subset of the deployed sensors for probing the environment allowing the conservation of the energy of the other sensors by turning off their signal processing circuitry. Based on the remaining energy at sensors, the gateway assigns the role of environment probing to the sensors with highest remaining energy. Knowing which sensors need to be active in signal processing, the gateway can dynamically adapt the network topology to reduce the energy consumed in communication, thus extending the life of the network while achieving acceptable performance for data transmission. To achieve this, the gateway must analyze sensor reports, monitor sensors heath status and above all control the operational mode of each sensor.

Our proposed architecture enables monitoring and controlling of sensor's operation. If the gateway cannot reach some sensors, they will operate continuously and deplete their energy rather quickly. In addition, redundant data traffic will cause collisions among the transmissions of different sensors and lead to unacceptable high rate of packet drop and waste significant sensor energy. Agent nodes will enable gateway 
commands to reach all sensors and prevent uncontrolled redundant data generation and transmission. The gateway will be able as well to instruct some sensors to switch to a low energy sleep mode and save energy. Moreover, agent nodes can further relay data from unreachable nodes to the gateway and thus enhance the coverage that the sensor network provides.

Monitoring potentially large number of sensors in such energy-constrained environment is challenging. Status updates can generate high communication traffic in such large network and can be an energy burden, especially if done frequently. On the other hand, the quality of the sensor organization and network management decisions can be negatively affected if based on wrong sensor status. Our approach calls for modeling and tracking consumption in communication and signal processing. The gateway's knowledge of active sensors and data routes enables the estimation of energy depletion at each sensor and thus limits the need for frequent status update.

Sensors within a group communicate only with their group leader (agent). There is single-hop routing within the group i.e. the agent and the group members communicate directly. Routing data from agents to the gateway use multi-hop paths. In our routing approach, we model the sensor network as a graph with agent sensors and gateway nodes that are connected by bi-directional wireless links with a cost associated with each direction. Given that the gateway organizes the sensors, it can combine the consideration for energy commitments to data processing, remaining sensor energy, link quality and sensor location in the link cost to achieve efficient setup of message routes. Each link may have a different cost for each direction due to different energy levels of the nodes at each end. The cost of a path between two nodes is defined as the sum of the costs of the links traversed. The routing decisions take into consideration the agent's load as a function of the inbound traffic.

The cost function for a link between nodes $i$ and $j$ is defined as follows:

$\sum_{k=0}^{7} C F_{k}=c_{0} \times\left(\text { distance }_{i j}\right)^{l}+c_{1} /$ energy $_{j}+c_{2} \times$ Active inbound links $+c_{3}+c_{4}$

Where: distance $_{i j}$ is the distance between the nodes $i$ and $j$, energy ${ }_{j}$ is the current energy of node $j$, and $C F_{k}$ are cost factors defined as follows:

$>C F_{0}$ : Communication cost $=c_{0} \times\left(\text { distance }_{i j}\right)^{l}$, where $c_{0}$ is a weighting constant and the parameter $l$ depends on the environment, and typically equals to 2 . This factor reflects the cost of the wireless transmission power, which is directly proportional to the distance raised to some power $l$.

$>C F_{l}$ : Energy stock $=c_{1} /$ energy $y_{j}$ for node $j$. This cost factor favors nodes with more energy. The more energy the node has, the better it is for routing.

$>C F_{2}$ : relaying load $=c_{2} \times$ number of inbound active link for node $j$. This factor extends the life of overloaded relay nodes by making them less favorable. Since these relay nodes are already critical by being on more than one path, avoiding overloading these nodes would increase the reliability of paths through them.

$>C F_{3}$ : Relay enabling cost $=c_{3}$, where $c_{3}$ is a constant reflecting the overhead required to switch an inactive node to become a relay. This factor makes the algorithm favors the relay-enabled nodes for routing rather than inactive nodes.

$>\mathrm{CF}_{4}$ : Sensing-state cost $=c_{4}$, where $c_{4}$ is a constant added when the node $j$ is in a sensing-sate. This factor does not favor selecting sensing-enabled nodes to serve as relays, since they have committed some energy for data processing. 
For each sensing-enabled node, we find a least-cost path from the agent of that node, or the node itself if it is not a member of a group, to the gateway using Djikstra's Shortest Path algorithm. Again a sensing-enabled node forwards its data to the agent using direct links. The typical operation of the network consists of three alternating cycles: status update cycle, routing cycle and data cycle. In the status update cycle every agent queries the status of its group members and sends along with its own status directly to the gateway. The status mainly reflects the remaining energy. According to the status of nodes, the gateway adjusts the use pattern or even stops counting on it if it fails. In case of an agent failure, the gateway performs a limited regrouping during which the group members of the failed agent are reassigned to other agents. During the routing cycle, the state of each node in the network is determined by the gateway based on the application and routing algorithm and the nodes are then informed about their newly assigned responsibilities and how to route the data. In the data cycle, the nodes, which are sensing the environment, send their data to the gateway over the designated paths.

\section{Experimental Validation}

The effectiveness of our approach is validated through simulation. This section describes performance metrics, simulation environment and experimental results. We use the following metrics to capture the performance of our approach:

- Node reachability: The number of sensors that can be reached by the gateway. It is an indication of the effectiveness of the architecture and the use of sensor grouping.

- Time for first node to die: Losing an active node typically trigger either reorganization or re-routing or both. This metric gives an indication of network lifetime since the network can get partitioned and become hard to manage.

- Average and standard deviation of node lifetime: This also gives a good measure of the network lifetime. An approach, which minimizes the standard deviation of node life, is predictable and thus desirable.

- Average energy consumed per packet: A routing algorithm that minimizes the energy consumed per packet will, in general, yields better energy savings.

\subsection{Environment Setup}

In the experiments a set of 100 sensors is randomly placed in a $1000 \times 1000 \mathrm{~m}^{2}$ area. The gateway is randomly positioned within the boundaries of the deployment region. A free space propagation channel model is assumed with the capacity set to $2 \mathrm{Mbps}$ [212]. Packet lengths are $10 \mathrm{Kbit}$ for data packets and $2 \mathrm{Kbit}$ for routing and status packets. Each node is assumed to have an initial energy of 5 joules and a buffer for up to 15 packets 22 . A node is considered non-functional if its energy level reaches 0 . Some links among sensors in close proximity and between the gateway and some sensors are randomly selected to be broken to simulate terrain effects. For a node in the sensing state, packets are generated at a constant rate of 1 packet/sec 8 . Each data packet is time-stamped when generated to allow tracking delays. In addition, each 
packet has an energy field that is updated during the packet transmission to calculate energy per packet. A packet drop probability is taken equal to 0.01 . This is used to make the environment more realistic and to simulate the deviation of the gateway energy model from the actual energy. The energy model of sensors is described in Appendix A.

We assume that the network is tasked with a target-tracking mission in the experiment. The initial set of sensing nodes is chosen to be the nodes on the convex hull of the deployed sensors. The set of sensing nodes change as targets move. Since targets are assumed to come from outside the deployment area, the sensing circuitry of all boundary nodes is always turned on. The sensing circuitry of other nodes are usually turned off but can be turned on according to targets movement. Targets are assumed to start at a random position outside the convex hull and move linearly through the deployment area. These targets are characterized by having a constant speed chosen uniformly from the range four to six $\mathrm{m} / \mathrm{sec}$ and a constant direction chosen uniformly depending on the initial target position in order for the target to cross the convex hull region.

\subsection{Simulation Results}

Experiments were run for 5 seed values varying the number of deployed sensors each time. The gateway's transmission range was taken as $500 \mathrm{~m}$ while the sensors' transmission range was set to $50 \mathrm{~m}$. The values chosen for $a_{1}$ and $a_{2}$ in the simulation are 0.6 and 0.4 , respectively. For the purpose of our simulation experiments the values for the parameters $\left\{c_{i}\right\}$ are initially picked based on sub-optimal heuristics for best possible performance. As mentioned earlier, some communication links among sensors in close proximity and between the gateway and sensors were broken to simulate the presence of obstacles. We compared the performance of our system with a system without grouping incorporated. The results (figures 5 through 8 ) show that the algorithm gives relatively good performance for all metrics especially as the number of deployed sensors is increased. We calculated the "Node Reachability" ratio as follows:

Node Reachability $=$ Number of reachable sensors (using grouping) / Total number of sensors.

The figures show that our algorithm performs better when the sensors are densely populated since the probability of an unreachable sensor finding an agent in its vicinity increases. Our approach outperforms a system without grouping by approximately a factor of $85 \%$ in terms of node reachability.

Also in the new system model the gateway receives periodic status updates from the sensors; hence it can manage the sensors and perform educated routing decisions thus increasing the average lifetime of the nodes by a factor of 1.28 over the other system. Since unreachable sensors need to communicate only to their one-hop agents and the load on agents is taken into consideration while routing, it leads to better energy savings. Hence our system model gives a marginal improvement of $9 \%$ over a system without any grouping in terms of average energy consumed per packet. 

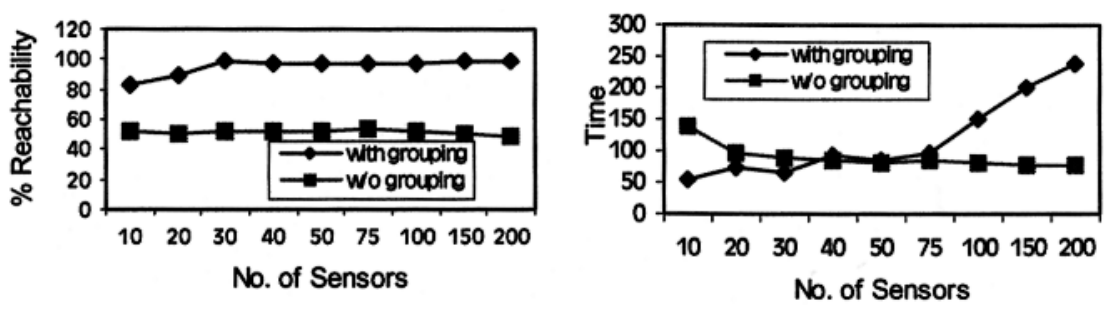

Fig. 5. Effect of grouping on node reachability

Fig. 6. Comparison of time to network partition

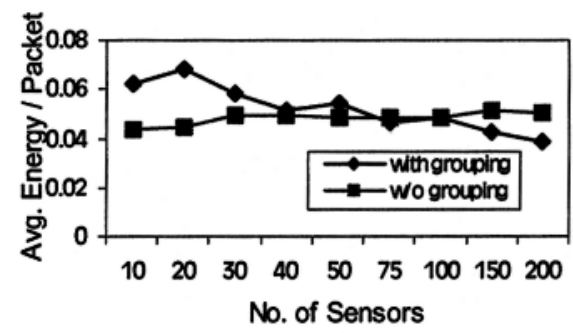

Fig. 7. Effect of grouping on average energy of Packet

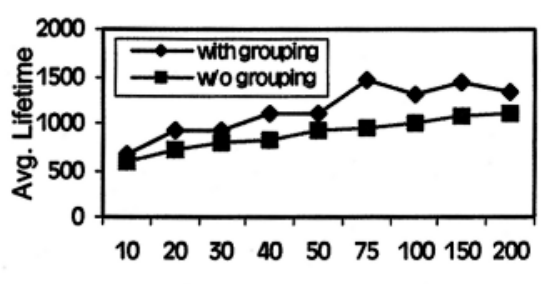

No. of Sensors

Fig. 8. Effect of grouping on average lifetime of node packet

\section{Conclusions}

Over the last few years, the design of unattended sensor networks has gained increasing importance due to their potential for some civil and military applications. Sensors are typically deployed randomly in an area of interest to probe the environment and transmit collected reports to a command center via radio. Due to terrain and high level of signal interference some sensors can be unreachable. In addition, sensors are usually battery-operated and their energy has to be managed wisely.

In this paper, we have introduced a novel approach for efficient monitoring and energy-aware management of wireless sensor networks. A less energy-constrained gateway node is used to manage network operation. A gateway node controls sensor functions, monitors sensor status and sets routes for sensor data. The gateway tracks energy usage at every sensor node and changes in the mission and the environment. Sensors are grouped to ensure that they can be reached via a member of its group. To limit monitoring traffic, energy consumption at the sensor is accurately modeled and tracked. Simulation results demonstrate that our architecture increases sensor reachability and the network lifetime along with better energy savings especially in densely populated sensor networks.

In the future we plan to extend the approach to address medium access issues related to the presented architecture, in particular time-based arbitration. In addition, we would like to investigate the problem of optimal placement of the gateway for enhanced sensor's reachability. 


\section{References}

1. Akyildiz, I.F., et al.: Wireless sensor networks: a survey, Computer Networks, Vol 38 (2002), 393-422.

2. Estrin, D., et al.: Next Century Challenges: Scalable Coordination in Sensor Networks, Proceedings of the Fifth Annual International Conference on Mobile Computing and Networks (MobiCOM '99), Seattle, Washington, August 1999

3. Pottie, G. J., Kaiser, W.J.: Wireless integrated network sensors, Communications of the ACM, Vol. 43, No 5 (2000) 51-58

4. Sohrabi, K., et al.: Protocols for self-organization of a wireless sensor network, IEEE Personal Communications, Vol. 7, No. 5 (2000) 16-27

5. Min, R., et al.: Low Power Wireless Sensor Networks, in the Proceedings of Internation Conference on VLSI Design, Bangalore, India, January 2001

6. Burne, R., et al: A Self-Organizing, Cooperative UGS Network for Target Tracking, Proceedings of the SPIE Conference on Unattended Ground Sensor Technologies and Applications II, Orlando Florida, April 2000

7. Meguerdichian, S., et al.: Localized Algorithms in Wireless Ad hoc Networks: Location Discovery and Sensor Exposure, Proceedings of the IEEE/ACM Workshop on Mobile Ad Hoc Networking and Computing (MobiHOC 2001), October 2001

8. Mathew, R, Younis, M.: Energy-Efficient Bootstrapping Protocol for Sensor Network, Proceedings of the International Conference on Wireless Networks (ICWN'02), Las Vegas, Nevada, June 2003

9. Data sheet for the Acoustic Ballistic Module, SenTech Inc., http://www.sentech-acoustic.com/

10. Intanagonwiwat, C., Govindan, R., Estrin, D: Directed diffusion: A scalable and robust communication paradigm for sensor networks, Proceedings of the Sixth Annual International Conference on Mobile Computing and Networking (MobiCOM '00), Boston, Massachussetts, August 2000

11. Heinzelman, W., Kulik, J., Balakrishnan, H.: Adaptive protocols for information dissemination in wireless sensor networks, Proc. Fifth Annual ACM/IEEE International Conference on Mobile Computing and Networking (MobiCom '99)

12. Heinzelman, W., Chandrakasan, A., Balakrishnan, H.: Energy-efficient communication protocol for wireless sensor networks, Proc. Hawaii Intl. Conf. System Sciences, Hawaii, (2000) 3005-3014

13. Shah, R., Rabaey, J.: Energy Aware Routing for Low Energy Ad Hoc Sensor Networks, Proceedings of the IEEE Wireless Communications and Networking Conference (WCNC'02), Orlando, FL, March 2002

14. Chen, B., et al: Span: an energy-efficient coordination algorithm for topology maintenance in ad-hoc wireless networks, Proceedings of MobiCom 2001, Rome, Italy, July 2001

15. Xu, Y., Heidemann, J., Estrin, D.: Geography-informed energy conservation for ad-hoc routing, Proceedings of MobiCom 2001, Rome, Italy, July 2001

16. Cerpa, A, Estrin, D.: ASCENT: adaptive self-configuring sensor networks topologies, Proceedings INFOCOM 2002, New York, June 2002

17. Hsin, C-f, Mingyan Liu: A Distributed Monitoring Mechanism for Wireless Sensor Networks, Technical Report, EECS Department, University of Michigan. Spring, 2002

18. Chessa, S., Santi, P.: Crash Faults Identification in Wireless Sensor Networks, Computer Communications, Vol. 25, No. 14 (2002) 1273-1282

19. Zhao, Y. J., Govindan, R., Estrin, D.: Residual Energy Scan for Monitoring Sensor Networks, Proceedings of the IEEE Wireless Communications and Networking Conference (WCNC'02), March 2002

20. Singh, S., Raghavendra, C.S.: PAMAS: power aware multi-access protocol with signaling for ad hoc networks, ACM Computer Communications Review, July 1998 
21. Arisha, K, Youssef, M., Younis, M.: Energy-Aware TDMA-Based MAC for Sensor Networks, Proceedings of the IEEE Workshop on Integrated Management of Power Aware Communications, Computing and Networking (IMPACCT 2002), New York City, New York, May 2002

22. Andresen, J.B, Rappaport, T.S., Yoshida, S.: Propagation Measurements and Models for Wireless Communications Channels, In IEEE Communications Magazine, Vol. 33, No. 1, (1995)

23. Gerla, M., Pei, G., Lee, S.J.: Wireless, Mobile Ad-Hoc Network Routing,, IEEE/ACM FOCUS 99, New Brunswick, NJ, May 1999

24. Bhardwaj, M., et. al : Upper Bounds on the Lifetime of Sensor Networks, Proceedings of ICC 2001, June 2001 (12)

25. Min, R., et. al : An Architecture for a Power-Aware Distributed Microsensor Node, IEEE Workshop on Signal Processing Systems (SiPS '00), October 2000

26. Heinzelman, W., et al: Energy-Scalable Algorithms and Protocols for Wireless Microsensor Networks, Proc. International Conference on Acoustics, Speech, and Signal Processing (ICASSP '00), June 2000

27. Sinha, A., Chandrakasan, A.: Energy Aware Software, Proceedings of the 13th International Conference on VLSI Design, Calcutta, India (January 2000) 50-55

\section{Appendix A: Sensor's Energy Consumption Model}

Since the presented routing algorithm uses model-based sensor energy consumption, it is important to use a fairly accurate model to ensure the effectiveness of the approach and minimize the correction made to the gateway-view of the sensor-energy level during the refresh phase. A typical sensor node consists mainly of a sensing circuit for signal conditioning and conversion, digital signal processor, and radio links 2324. The following summarizes the energy-consumption models for each sensor component.

\section{Communication Energy Dissipation}

We use the model of 112325 . The key energy parameters for communication in this model are the energy/bit consumed by the transmitter electronics $\left(\alpha_{11}\right)$, energy dissipated in the transmit op-amp $\left(\alpha_{2}\right)$, and energy/bit consumed by the receiver electronics $\left(\alpha_{12}\right)$. Assuming a $1 / \mathrm{d}^{\mathrm{n}}$ path loss, the energy consumed is:

$$
E_{t x}=\left(\alpha_{11}+\alpha_{2} d^{n}\right) * r \text { and } E_{r x}=\alpha_{12} * r
$$

Where $E_{t x}$ is the energy to send $r$ bits and $E_{r}$ is the energy consumed to receive $r$ bits. Table 1 summarizes the meaning of each term and its typical value.

\section{Computation Energy Dissipation}

We assume the leakage current model of 242526. The model depends on the total capacitance switched by the program and the number of cycles the program takes. We used parameter values similar to those in 26. 
Table 1. Parameters for the communication energy model

\begin{tabular}{|c|l|}
\hline Term & \multicolumn{1}{c|}{ Meaning } \\
\hline$\alpha_{11}, \alpha_{12}$ & $\begin{array}{l}\text { Energy dissipated in transmitter and receiver electronics per bit } \\
\text { (Taken to be } 50 \mathrm{~nJ} / \mathrm{bit}) .\end{array}$ \\
\hline$\alpha_{2}$ & Energy dissipated in transmitter amplifier $\left(\right.$ Taken $=100 \mathrm{pJ} / \mathrm{bit} / \mathrm{m}^{2}$. \\
\hline$r$ & Number of bits in the message. \\
\hline$d$ & Distance that the message traverses. \\
\hline
\end{tabular}

\section{Sensing Energy Dissipation}

We assume that the energy needed to sense one bit is a constant $\left(\alpha_{3}\right)$ so that the total energy dissipated in sensing $r$ bits is 23: $E_{\text {sensing }}=\alpha_{3} * r$

For the Ballistic Audio Module sensor 8, the energy dissipated for sensing a bit is approximately equal to the energy dissipated in receiving a bit. Therefore, the parameter $\alpha_{3}$ is taken equal to $\alpha_{12}$. 